\title{
Second Parameter Effects in and between M3 and Palomar 3
}

\author{
M. Catelan, R. T. Rood \\ University of Virginia, Department of Astronomy, P.O. Box 3818, \\ Charlottesville, VA 22903-0818, USA \\ F. R. Ferraro \\ Osservatorio Astronomico di Bologna, via Ranzani 1, I-40126 Bologna, \\ Italy
}

\begin{abstract}
We study the globular clusters M3 and Palomar 3 as a "second parameter (2ndP) pair," showing that: i) M3 has a surprisingly strong internal 2ndP; ii) The dispersion in mass on the Pal 3 horizontal branch (HB) is intrinsically very small, leading to the most apparent differences in HB morphology between M3 and Pal 3; iii) Ignoring the difference in $\mathrm{HB}$ mass dispersion between M3 and Pal 3, their relative HB types can be accounted for by a fairly small difference in age, of order $0.5-1$ Gyr.
\end{abstract}

\section{Internal Second Parameter in M3}

Figure 1 shows the M3 HB CMD for the innermost $\left(r<50^{\prime \prime}\right.$, upper panel) and for the outermost $\left(r>210^{\prime \prime}\right.$, lower panel) cluster regions. The numbers of red ("R"), RR Lyrae variable ("V") and blue ("B") HB stars are indicated. It is apparent that the innermost regions of M3 have a much bluer HB than the outermost ones. Even though the inner regions were observed with HST-WFPC2 while the outermost regions were observed from the ground, we emphasize that the difference is real, extremely significant, and cannot be accounted for by any conceivable source of systematic observational error.

\section{An Intrinsically Small Mass Dispersion on the Pal 3 ZAHB}

The HB region of the CMD of Pal 3 is shown in Fig. 2. This is essentially the same as the Stetson et al. (1999) HST-WFPC2 CMD, except that here the individual RR Lyrae variables are plotted, based on mean colors and magnitudes derived by combining the HST photometry and the Borissova et al. (1998) ground-based photometry. Note that all cluster RR Lyraes are ab-type variables, and there are no blue-HB stars. A theoretical ZAHB and evolutionary track are overplotted. The single track spans the entire observed HB showing that little (if any) intrinsic dispersion in mass on the ZAHB is needed to account for the Pal $3 \mathrm{HB}$ morphology. Extensive Monte Carlo simulations confirm this. 


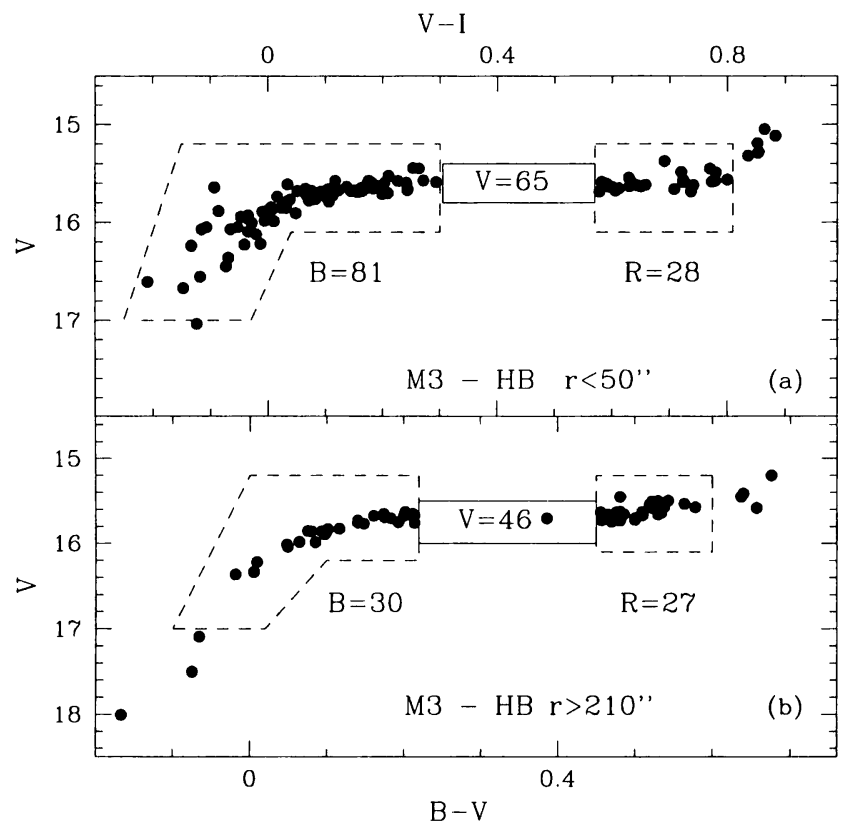

Figure 1. M3, once thought to be the prototypical "canonical" globular cluster, shows a very strong internal second parameter, with a much bluer HB morphology in its inner regions (upper diagram) than in its outskirts (lower diagram).

\section{The Difference in Age between M3 and Pal 3}

Ignoring, for the sake of argument, the internal 2ndP in M3 and the difference in mass dispersion between the M3 and Pal $3 \mathrm{HBs,} \mathrm{we} \mathrm{find,} \mathrm{using} \mathrm{an} \mathrm{approach}$ similar to Catelan's (2000), that the relative HB types of M3 and Pal 3 can be easily accounted for by a difference in age of $\approx 0.5-1$ Gyr (Fig. 3), as indeed favored by VandenBerg (2000) from analysis of the clusters' turnoffs. The larger age difference favored by Stetson et al. (1999), $\approx 2 \mathrm{Gyr}$, is not consistent with the relative $\mathrm{HB}$ types of the clusters under the assumption that this is a "bona fide" 2ndP pair.

\section{References}

Borissova, J., Spassova, N., Catelan, M., \& Ivanov, V. D. 1998, in ASP Conf. Ser. Vol. 135, A Half-Century of Stellar Pulsation Interpretations, ed. P. A. Bradley \& J. A. Guzik (San Francisco: ASP), 188

Catelan, M. 2000, ApJ, 531, 826

Stetson, P. B., et al. 1999, AJ, 117, 247

VandenBerg, D. A. 2000, ApJS, 129, 315 


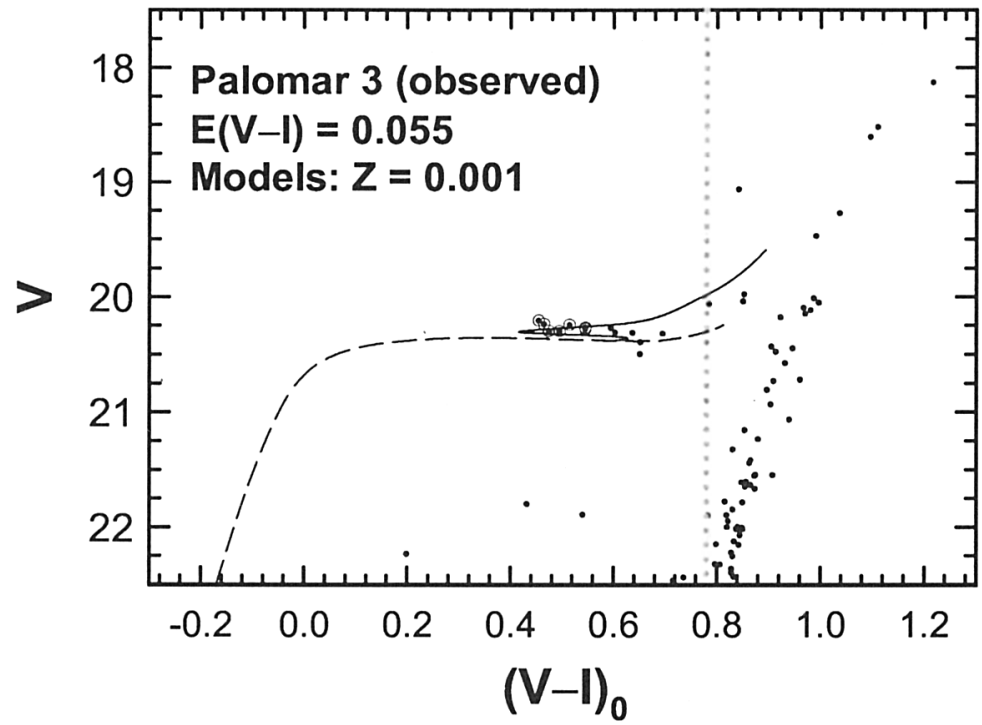

Figure 2. The CMD of Pal 3, with individual $\mathrm{RR}$ Lyrae variables plotted. A ZAHB and evolutionary track for $Z=0.001$ are overplotted. The vertical dotted line shows the mean color of the Pal 4/Eridanus HBs. The HB morphology of Pal 3 can be accounted for without the need to assume a significant dispersion in mass on the ZAHB.

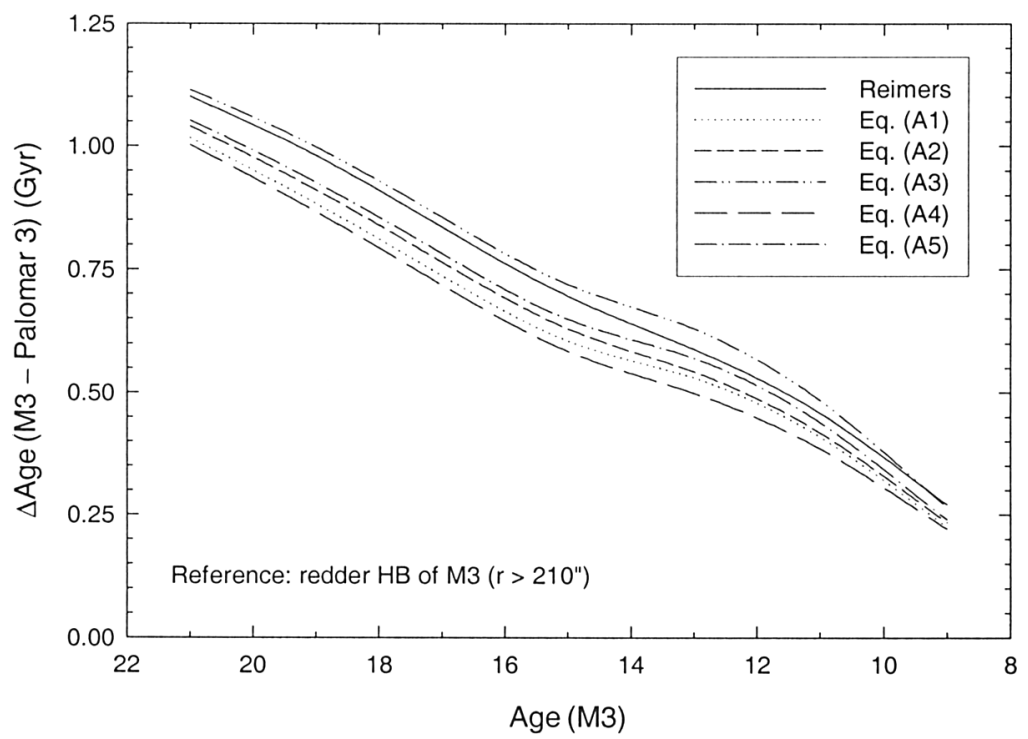

Figure 3. Relative HB morphology ages for M3 vs. Pal 3, plotted as a function of the absolute $\mathrm{M} 3$ age. This assumes the outermost regions of M3 to be more representative of the cluster as a whole. The lines correspond to different mass loss formulae for giants (Catelan 2000). 